\title{
Electron spin resonance investigation of electronic states in hydrogenated microcrystalline silicon
}

\author{
J. Müller, F. Finger, R. Carius, and H. Wagner \\ Forschungszentrum Jülich, ISI-PV, 52425 Jülich, Germany
}

(Received 28 January 1999)

\begin{abstract}
Phosphorus-doped microcrystalline silicon with high-crystalline volume fraction was prepared by very highfrequency plasma enhanced chemical vapor deposition. The material is studied by electron spin resonance and transport measurements as a function of doping and temperature. In all samples a resonance at $g=1.998$ is found with spin densities very similar to the phosphorus dopant density and also the carrier density at high doping levels. This resonance is related to doping-induced excess electrons. Its spin density is largely temperature independent, and the corresponding electrons occupy dopant or conduction band tail states at low temperatures, while they are excited into the conduction band at high $T$. This gradual transition is accompanied by changes in linewidth, $g$ value and spin-lattice relaxation time. Hyperfine interaction with $P$ nuclei is only observed for intermediate doping levels and has very small intensity. From the value of the hyperfine splitting, the effective Bohr radius of the impurity wave function is estimated to $12 \AA$. Transport at low temperatures $(T<20 \mathrm{~K})$ proceeds via hopping between donor states and/or conduction-band tail states. A thermal activation energy of $3.5 \mathrm{meV}$ and similar localization lengths as from the hyperfine data are found for this process. At temperatures above $20 \mathrm{~K}$ electronic transport is governed by a wide distribution of activation energies.
\end{abstract}

[S0163-1829(99)00239-8]

\section{INTRODUCTION}

Hydrogenated microcrystalline silicon ( $\mu c-\mathrm{Si}: \mathrm{H})$ prepared by plasma-enhanced chemical vapor deposition (PECVD) is a material already used routinely as highly conductive contact layer in thin-film solar cells where the active layers are made of amorphous silicon $(a-\mathrm{Si}: \mathrm{H})$ or its alloys. Recently effort has been made with considerable success to use $\mu c$-Si:H prepared by low-temperature processes like PECVD also as active layer to replace the $a$-Si:H alloy materials. ${ }^{1,2}$ This has initiated renewed research activities of both the technological aspects of material preparation and the electronic properties of $\mu c-\mathrm{Si}: \mathrm{H}-$ in particular investigations of those aspects that might affect the photovoltaic performance. Structural investigations of $\mu c$-Si:H (Refs. 3 and 4) show that the material consists of areas (grains) of perfect crystallinity with an average size of $20 \mathrm{~nm}$ forming larger columns parallel to the film growth axis and extending over the entire film thickness. Between these columns one finds disordered regions or internal voids, and the individual small "grains" are separated from each other only by crystalline imperfections like stacking faults.

As a result of the mixed-phase nature of microcrystalline silicon specific information about a particular electronic state can only be gained by experimental techniques that are sensitive to the microscopic environment of this state. One technique which fulfills this requirement is electron spin resonance (ESR).

In previous ESR studies, we found three resonances in microcrystalline silicon. Two of them are attributed to defect states and are found in intrinsic as well as in doped material. The respective $g$ values are $g=2.0043$ and $g=2.0052$. The latter resonance is attributed to $\mathrm{Si}$ dangling-bond (DB) states. ${ }^{5-8}$ The resonance at $g=2.0043$ cannot be unambiguously identified. From its resonance parameters it is probably related to dangling-bond states in $\mathrm{Si}$-rich $\mathrm{Si}-\mathrm{O}$ layers, ${ }^{9,10}$ as a strong oxygen take up has been observed in secondary ion mass spectroscopy (SIMS) measurements on $\mu c-\mathrm{Si}: \mathrm{H}$ films, ${ }^{11}$ and an increase of a signal at $g=2.0044$ in $\mu c-\mathrm{Si}: \mathrm{H}$ samples that were exposed to air for several weeks has been reported. $^{12}$

In $n$-type material or under light illumination a third resonance appears at $1.997<g<1.998$, which is attributed to conduction electrons in the crystalline grains and is referred to as conduction electron $(\mathrm{CE})$ resonance. A close correspondence to the conduction electron resonance in crystalline silicon $(c-\mathrm{Si})$ is exhibited by a similar $g$ value as in $c$ - $\mathrm{Si}, 9,13,14$ an increase in linewidth between 20 and $300 \mathrm{~K}$ (Ref. 15) like in crystalline silicon ${ }^{13,16}$ and the short spin-lattice relaxation times $T_{1}$ as measured by pulsed ESR techniques, ${ }^{17,18}$ which are also similar to $T_{1}$ times reported for $c$-Si. ${ }^{19-22}$

For slightly doped $n$-type microcrystalline silicon samples and temperatures lower than $150 \mathrm{~K}$ the signal intensity of the $\mathrm{CE}$ resonance changes with temperature proportional to $1 / T$ (Curie-law), ${ }^{5,15}$ which is equivalent to a constant CE spin density. However, the nature and the energy position of such states have been unclear so far.

It was further found that the doping induced Fermi-level shift leads to a steady increase of both CE spin density and room-temperature dark conductivity $\left(\sigma_{\mathrm{RT}}\right) .{ }^{15,18,23,24}$ To interpret this behavior a quantitative comparison of the $\mathrm{CE}$ spin density with carrier densities obtained by techniques other than ESR is essential.

In the present paper, we concentrate on the investigation of the CE resonance associated with states within the crystalline regions of the material, which make up more than $90 \%$ of the sample volume. Therefore, a detailed comparison of $\mu c-\mathrm{Si}: \mathrm{H}$ with the large amount of data available for crystalline silicon is necessary. Signals attributed to (i) electrons located at phosphorus donors, ${ }^{25,26}$ (ii) electrons in clusters of two or more dopant atoms ${ }^{16,21,27}$ and (iii) electrons in conduction or impurity band states $9,13,28,29$ are found in crystalline material, where a distinction between the different kinds 
of states is often difficult. It is the aim of this study to establish a clear relationship between ESR and transport measurements in $n$-type microcrystalline silicon, to identify the origin of the $\mathrm{CE}$ resonance in the different temperature and doping regimes and to interpret the observed changes in spin density, $g$ value and linewidth with regard to this identification.

The outline of the paper is as follows: The experimental procedures are explained in Sec. II. In Sec. III, the structure of $\mu c-\mathrm{Si}: \mathrm{H}$ is described, we comment on the consequences of this structure on the electronic states and finally recall some basic relations on paramagnetic susceptibility and hyperfine interaction. In Sec. IV the doping and temperature dependence of the CE resonance (with respect to $g$ value, intensity, linewidth, and hyperfine interaction) as well as temperature-dependent electrical conductivity measurements are presented. The results are discussed in Sec. V with emphasis on the relationship between ESR and transport, its implications on the origin of the CE line and a comparison with crystalline silicon. We conclude in Sec. VI.

\section{EXPERIMENTAL PROCEDURES}

Microcrystalline silicon samples were prepared by very high-frequency plasma enhanced chemical vapor deposition in a diode-type reactor from mixtures of silane and hydrogen with a constant gas flow ratio of $\left[\mathrm{SiH}_{4}\right]:\left[\mathrm{H}_{2}\right]=3: 97$. For controlled low-level $n$-type doping various amounts of $\mathrm{PH}_{3}$ were added. The substrate temperature was $200^{\circ} \mathrm{C}$, the discharge power $5 \mathrm{~W}$ and the plasma excitation frequencies $\mathrm{v}_{\mathrm{ex}}$ and total gas pressure $p$ were $95 \mathrm{MHz}$ and $300 \mathrm{mTorr}$, respectively, except for one sample with $\mathrm{v}_{\mathrm{ex}}=115 \mathrm{MHz}$ and $p=150 \mathrm{mTorr}$. These deposition parameters resulted in $\mu c$ material with average sizes of $20 \mathrm{~nm}$ for the "unperturbed" grains determined from x-ray diffraction (XRD) measurements and high-crystalline volume fractions of more than 90\% as estimated by Raman and confirmed by transmission electron microscopy (TEM) and XRD. We varied the gas phase doping ratio, i.e., the gas flow ratio $\left[\mathrm{PH}_{3}\right]:\left[\mathrm{SiH}_{4}\right]$, between 1 and $133 \mathrm{ppm}$ obtaining room-temperature conductivities between $2.7 \times 10^{-4} \mathrm{~S} / \mathrm{cm}$ and $5.1 \times 10^{-1} \mathrm{~S} / \mathrm{cm}$.

We deposited samples on aluminum foil and on roughened (to avoid peeling off of films) quartz or borosilicate glass in the same run to have material for both ESR and conductivity measurements. The thickness of these films was between 2 and $3 \mu \mathrm{m}$. The aluminum foil was etched away by $\mathrm{HCl}$ after deposition yielding between 30 and $60 \mathrm{mg}$ of powder material for ESR measurements. The powder is sealed under $\mathrm{He}$ atmosphere in quartz tubes to maintain a defined environment after the sealing. As no general difference between ESR results of thin films on glass substrates and powdered samples was detected, most of the ESR measurements could be performed on powdered material due to the higher intensity and better signal-to-noise ratio.

Continuous-wave ESR measurements were performed with a commercial X-band spectrometer (Bruker ESP 380E) and a cylindrical transverse magnetic $\left(\mathrm{TM}_{110}\right)$ or a rectangular transverse electric $\left(\mathrm{TE}_{301}\right)$ cavity in the temperature range between 4.5 and $300 \mathrm{~K}$ with $100 \mathrm{kHz}$ modulation frequency and a modulation amplitude of 2-4 G. For measurements of the hyperfine interaction a modulation amplitude of $14 \mathrm{G}$ was used to enhance the broad hyperfine lines $\left(\Delta H_{p p}=25\right.$ $-35 \mathrm{G})$ relative to the center line. In order to obtain reliable spin densities for both the $\mathrm{CE}$ and the defect resonances at a measurement temperature of $40 \mathrm{~K}$ (which turned out to be convenient for this purpose) the microwave power used was $7 \mu \mathrm{W}$, a power level at which none of the observed lines shows saturation effects. However, as we are mainly concerned with the CE line in this study, a higher microwave power was sometimes applied, at which the defect state resonances already strongly saturate [due to their longer spinlattice relaxation times $T_{1}$ (Ref. 17)] and the CE line could be distinguished more easily.

Samples were mounted in a conventional $\mathrm{He}$ gas flow cryostat (Oxford ESR 900) approximately $1 \mathrm{~cm}$ above the gas outlet and the AuFe/Chromel-thermocouple, which is used to measure the temperature. This leads to a small difference between the temperature reading and the sample temperature (the real sample temperature is expected to be slightly higher). The difference was measured, independent of the thermocouple calibration, by comparing the temperature reading with the temperature calculated from the line intensity of our standard sample (see below), which exhibits a Curie-like temperature dependence, relative to its room temperature value. A maximum deviation of $15 \mathrm{~K}$ in the intermediate temperature range $(200 \mathrm{~K})$ is found. For lower temperatures and near room temperature the difference is much less. Also the relative temperature deviation when measuring different samples at the same temperature reading is much smaller.

For a quantitative analysis ( $g$ value, spin density) we compare the ESR intensities of our samples with an unhydrogenated sputtered amorphous silicon sample on quartz as a secondary standard, which has been calibrated versus 1,1-diphenyl-2-picrylhydrazil. ${ }^{24}$ The intensity of the ESR signal was obtained as the absorption curve area by a numerical integration of the data. In cases where more than one line was visible, as in lightly $n$-doped samples at low microwave power, numerical curve fitting procedures had to be employed to obtain the intensities of the individual resonances. More details will be given in Sec. IV.

Secondary ion mass spectroscopy (SIMS) measurements of phosphorus concentrations were performed on the films on aluminum foil before etching away the metal. High-mass resolution mode was used to separate the $P$ signal from the interfering silicon hydride ions. Conductivity of films was measured with coplanar evaporated silver contacts in a continuous flow He cryostat. The error due to the roughened surface is less than a factor of two. Hall measurements were done in a six-point geometry.

Throughout the paper, we will use the following notation: every sample is simply denoted by its gas-phase doping ratio (1-133 ppm), e.g., $67 \mathrm{ppm}$ for sample 67.

\section{BACKGROUND}

\section{A. Structure and electronic states}

The complex, mixed phase structure of the material has been investigated by transmission electron microscopy (TEM) and x-ray diffraction (XRD) experiments. ${ }^{3,4}$ From XRD average sizes of perfect, unperturbed crystallites of 20 $\mathrm{nm}$ are deduced, which form "clusters" of a typical colum- 
nar shape perpendicular to the substrate. These clusters extend throughout the entire film thereby comprising $90 \%$ of the sample volume. Within the clusters only typical crystalline imperfections like stacking faults and twinning are present between adjacent grains, which are not expected to lead to paramagnetically active deep defect states. On the other hand, the disordered regions separating grain columns are likely to have a much higher density of point defects (dangling bonds).

While not containing many point defects, structural disorder will lead to the formation of localized band tail states in the 20-nm grains. Exponential band tails, extending several $100 \mathrm{meV}$ from the band edges into the gap were found for example in fine-grained polycrystalline silicon films ${ }^{30,31,32}$ and were attributed to small wavelength potential fluctuations due to the spatial disorder. ${ }^{33}$ In $a$-Si:H such states extend from the band edges into the gap over an energy range of $200 \mathrm{meV}$ (conduction-band tail) or $300-400 \mathrm{meV}$ (valence-band tail) (see, e.g., Ref. 34).

Hence, excess electrons in phosphorus doped $\mu c-\mathrm{Si}: \mathrm{H}$ may occupy different electronic states (like donor, conduction-band or conduction-band tail states), whose respective occupation is expected to be temperature dependent.

\section{B. Magnetic susceptibility in semiconductors}

As in an ESR experiment the integrated absorption intensity is proportional to the static susceptibility of the sample, we will not distinguish between ESR signal intensity and susceptibility but use both terms as synonyms. There are two cases of electronic paramagnetism referred to as Curie-like or Pauli-like behavior.

The paramagnetic susceptibility $\chi$ of a system of ideal noninteracting paramagnetic centers (spin $S=\frac{1}{2}$ and $g$ value $g \approx 2$ ), whose statistics are governed by the MaxwellBoltzmann distribution and whose total number is constant in the temperature range under question, shows a typical $1 / T$ - or Curie-like behavior (see, e.g., Ref. 35) according to

$$
\chi_{\text {Curie }}=\frac{n \cdot \mu_{0} \cdot \mu_{B}^{2}}{k T},
$$

where $n$ is the density of the paramagnetic centers, $\mu_{B}$ the Bohr magneton, $\mu_{0}$ the permeability of the vacuum, $k$ the Boltzmann constant, and $T$ the absolute temperature.

Equation (1) is also valid for electrons located at their donor sites in an $n$-doped semiconductor ${ }^{28}$ and for electrons excited into the conduction (or an impurity) band as long as their concentration remains low and the temperature high enough for the electron gas to be nondegenerate.

On the other hand, the susceptibility of a gas of noninteracting degenerate electrons at sufficiently low temperatures is $T$ independent (Pauli paramagnetism) and has the value ${ }^{36}$

$$
\chi_{\text {Pauli }}=\frac{n \cdot \mu_{0} \cdot \mu_{B}^{2}}{E_{F}} .
$$

Measurements of the temperature dependence of the susceptibility should, therefore, enable us to distinguish between the degenerate and the nondegenerate case. However, a distinction between localized and delocalized electrons is not possible from the temperature dependence of $\chi$ alone.

\section{Hyperfine interaction}

Electrons located at their donor atoms will in general interact with the magnetic moment of the phosphorus nucleus $\left({ }^{31} \mathrm{P}, 100 \%\right.$ natural abundance, nuclear spin $\left.I=\frac{1}{2}\right)$ giving rise to hyperfine (hf) splitting. In a cubic lattice with fourfold coordination, like in the crystallites of $\mu c-\mathrm{Si}: \mathrm{H}$, the anisotropic or dipolar part of the hyperfine interaction vanishes for symmetry reasons. The residual isotropic hyperfine interaction $A$ is of the Fermi contact form ${ }^{37}$

$$
A=\frac{2 \mu_{0}}{3} \cdot g_{e} \cdot g_{n} \cdot \mu_{B} \cdot \mu_{n} \cdot\left|\psi_{e}(r=0)\right|^{2},
$$

where $g_{e}$ and $g_{n}$ are the electronic and nuclear $g$ values, $\mu_{B}$ and $\mu_{n}$ are the Bohr and nuclear magneton, and $\Psi_{e}(r=0)$ is the value of the $s$-like electronic donor wave function at the position of the $\mathrm{P}$ nucleus. The strength of the hyperfine interaction is directly related to the localization of the dopant state electrons and can be used to estimate the localization length.

In crystalline silicon at low temperatures $(T<30 \mathrm{~K})$ and low-dopant concentrations $N_{d}\left(N_{d}<8 \times 10^{16} \mathrm{~cm}^{-3}\right)$ the majority of the electrons will be in the ground state of the donor atom. ${ }^{38}$ The sixfold degenerate ground state of phosphorus splits into a singlet state $A_{1}$, a doublet state $E$ and a triplet state $T_{2}$, the latter five states lying very close to each other and being separated from the singlet ground state $A_{1}$ by about $11 \mathrm{meV} \cdot{ }^{38,39}$ Only the wave function of the $A_{1}$ state has a nonvanishing amplitude at the nucleus and gives rise to hyperfine interaction resulting in the ESR observation of two lines. ${ }^{25,38}$ For higher temperatures, however, an increasing part of the electrons is activated into excited states or even conduction-band states with no hyperfine interaction and the hyperfine lines broaden as a result of exchange scattering between donor and conduction-band electrons. With increasing donor concentration the donors form clusters of two or more donor atoms leading to additional features in the resonance spectrum and also the gradual disappearance of the $\mathrm{hf}$ line pair. ${ }^{21,26,27,40-42}$ For even higher concentrations an increasing number of electrons occupy delocalized states in the conduction band or an impurity band. ${ }^{28,29}$ For a recent work on ESR in phosphorus-doped silicon see also Ref. 43.

In summary, hf interaction in $c$-Si is easiest observable in samples with low dopant concentration at low temperatures, and the same behavior might be expected for $\mu c-\mathrm{Si}: \mathrm{H}$. Interestingly enough, this is not the case as will be shown below (Sec. IV E), which has important consequences for the location and identification of the corresponding electronic states.

\section{RESULTS}

\section{A. General features of the spectra}

Figure 1 shows the ESR spectrum of sample 1 obtained at a temperature of $40 \mathrm{~K}$ in the dark (thick-solid line). Numerical fits for the two defect resonances (at $g=2.0052 / 2.0043$, Gaussian lines) and the CE resonance (at $g=1.998$, Lorentzian line) as well as the sum of the three fit curves (thin-solid line) are included.

The line shape of the CE resonance is asymmetric for the lowest doping levels but approaches a symmetrical Lorentz- 


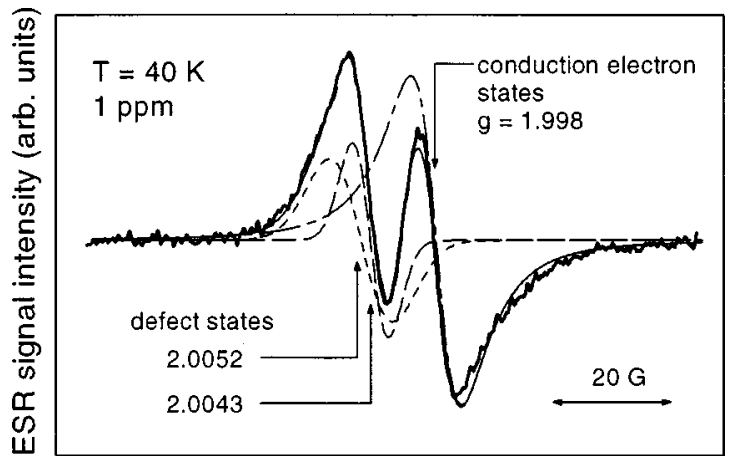

FIG. 1. Dark CW ESR spectrum of sample 1 at $40 \mathrm{~K}$. Included are numerical deconvolutions of the three superimposed resonances into two Gaussians (dashed lines) and one Lorentzian (dash-dotted line) as well as their sum (thin solid line).

ian line shape for higher doping. The origin of the asymmetric line shape is still unclear. In crystalline silicon with sufficiently high doping level and a sample thickness large compared to the skin depth the line shape of the CE resonance is asymmetric (Dysonian), ${ }^{44,45}$ while for powdered specimens with particle sizes of not more than several microns the line shape is Lorentzian. ${ }^{13,14,46}$ Taking into account the conductivities and the dimensions of the $\mu c-\mathrm{Si}: \mathrm{H}$ samples, a Dysonian line shape effect can be excluded. ${ }^{18}$ In order to compare resonance intensities without additional errors due to varying line-shape functions, we adopt the Lorentzian line shape for numerical fits of the CE line in all of our samples.

The line shapes of the defect state resonances could be simulated very well by Gaussian functions, keeping the resonance positions fixed at the respective $g$ values (2.0043, 2.0052) and sometimes also fixing the linewidth $\left(\Delta H_{p p}\right)$ of the Si-DB resonance at $12 \mathrm{G}$ (a value obtained from unrestricted fits in intrinsic and $p$-type samples over a wide temperature range ${ }^{47}$ ).

Figure 2 is a stack plot of dark ESR spectra for the samples investigated in this study. The spectra are normalized to the same peak-to-peak intensities. The defect state and $\mathrm{CE}$ resonances can be easily distinguished in the samples with lower doping levels as shown in detail in Fig. 1. At higher doping level the $\mathrm{CE}$ spin density $N_{S}(\mathrm{CE})$ strongly increases, whereas the defect signals remain around $2 \times 10^{16} \mathrm{~cm}^{-3}$ almost independent of doping. ${ }^{15,18}$ Therefore, the resonances of the DB lines become less visible with rising doping level.

\section{B. $g$ value}

In Fig. 3, the CE $g$ values obtained at a temperature of 40 $\mathrm{K}$ are plotted versus room-temperature conductivity $\sigma_{\mathrm{RT}}$. Included are samples without intentional phosphorus doping on the left-hand side of the figure (denoted by " $\langle i\rangle$ '), as some of those also exhibit a CE resonance in the dark. For the doped samples the CE $g$ values are around 1.9980 at doping levels below $33 \mathrm{ppm}$ and decrease continuously for higher doping levels to 1.9972 at $133 \mathrm{ppm}$. The undoped samples have $g$ values of 1.9980-85 exhibiting a large scatter due to the overlap with the defect resonances and the fact that the CE line appears to be more asymmetric for undoped material.

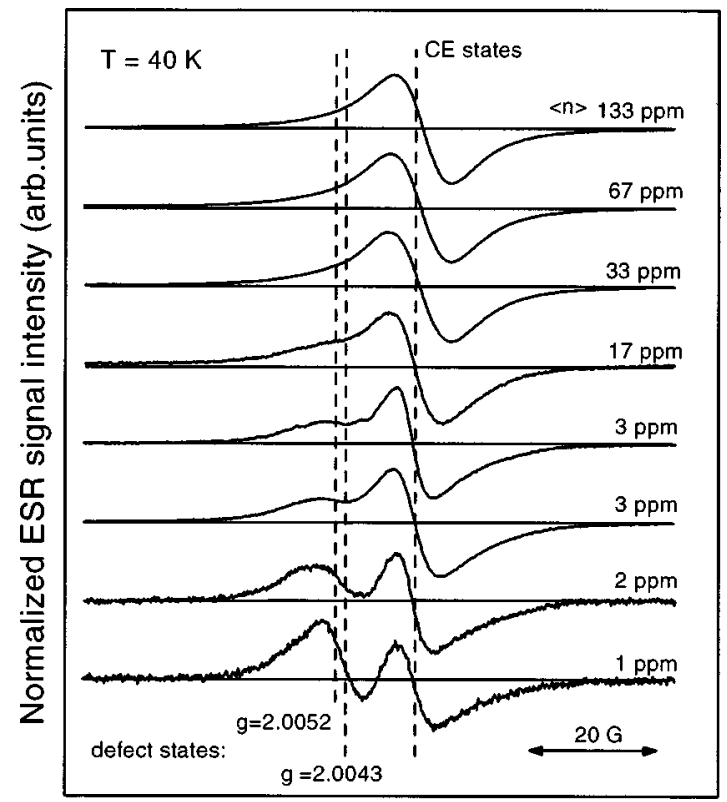

FIG. 2. Stack plot of dark CW ESR spectra for samples 1 to 133 at $40 \mathrm{~K}$. The spectra are normalized to the same peak-to-peak intensity.

Figure 4 shows the temperature dependence of the CE $g$ values for the $n$-type samples between 4.5 and $300 \mathrm{~K}$. For better readability error bars are only included at some points and the vertical bars without data markers (in the left part of the figure) represent the error margins for the respective samples at $T<100 \mathrm{~K}$. The $g$ values decrease with rising temperature.

\section{Linewidth}

The peak-to-peak linewidths $\Delta H_{p p}$ of the CE resonance for samples 1,3 , and 133 as a function of temperature between 4.5 and $300 \mathrm{~K}$ are compared in Fig. 5. Samples 1 and 3 on the one hand and sample 133 on the other hand are representative for material with low/intermediate and high doping levels, respectively. The errors in determining the linewidth are larger at high temperature, as background and

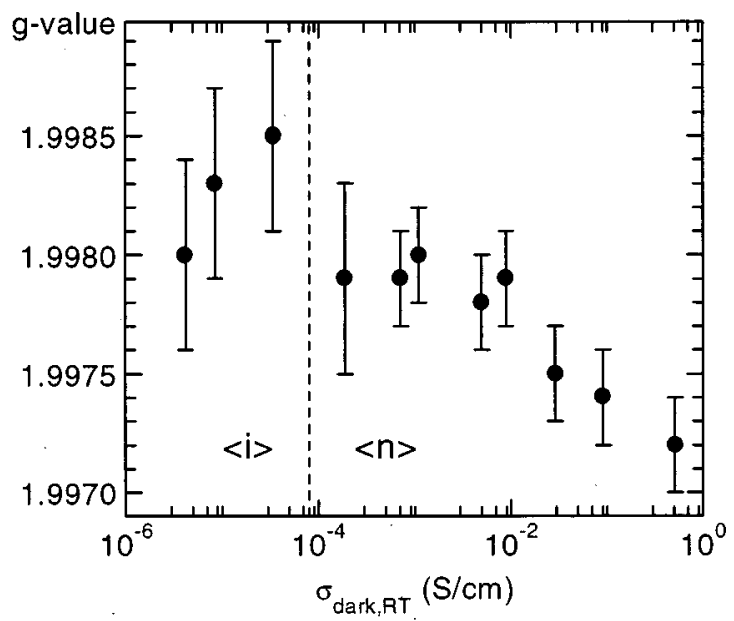

FIG. 3. CE $g$ values for intrinsic $(\langle i\rangle)$ and $n$-doped $(\langle n\rangle) \quad \mu c-\mathrm{Si}: \mathrm{H}$ as a function of room-temperature dark conductivity. 


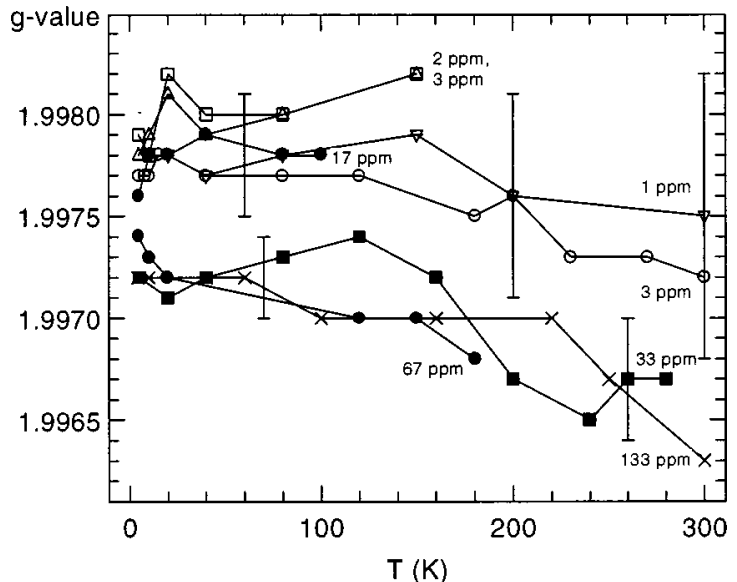

FIG. 4. CE $g$ values as a function of temperature. The vertical bars without data markers (in the left part of the figure) represent the error margins for the respective samples at $T<100 \mathrm{~K}$.

baseline contributions increasingly disturb the spectra. Above $30 \mathrm{~K}$ all samples show a large increase in the CE linewidth with rising $T$ and rising doping level. Below $30 \mathrm{~K}$ the $\Delta H_{p p}$-vs- $T$ curves flatten out, but increase again at the lowest temperatures for the samples 33, 67, and 133. For lower doping no such increase is observed.

\section{Susceptibility and spin density}

The temperature dependence of the CE signal intensity is shown in Fig. 6 for samples 1, 33, 67, and 133 in a doublelogarithmic plot. The straight line denotes the temperature dependence expected for Curie-like behavior, i.e., $\chi \propto 1 / T$.

Except for sample 1, observation of the CE line at higher $T$ is complicated by the fact that with rising temperature the conductivity of the samples increases. This leads to increasing dielectric losses inside the resonant cavity thus strongly reducing the quality factor $(\mathrm{Q})$ of the cavity. If the dielectric losses become too large, ESR measurements are no longer possible, as was the case for sample 67 , which could only be measured up to $180 \mathrm{~K}$. Since the signal intensity is proportional to the cavity quality factor, a decrease in $\mathrm{Q}$ also leads to a decrease in signal intensity. The CE intensities were

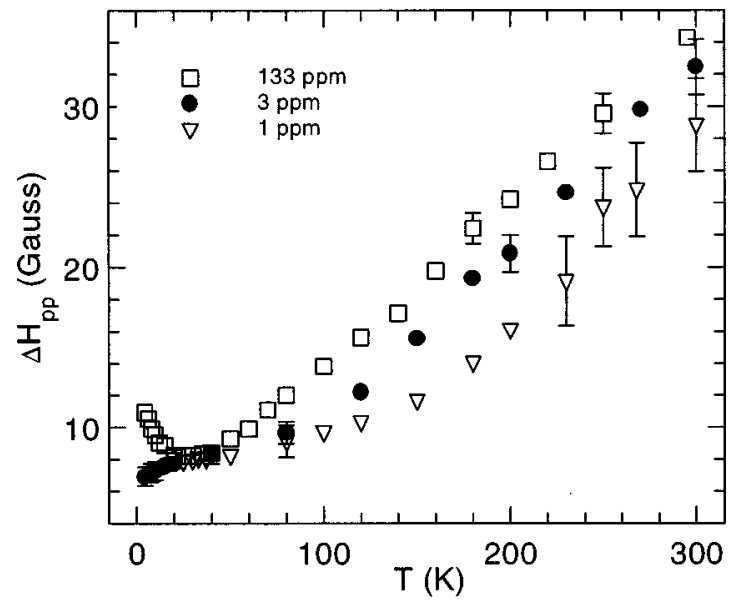

FIG. 5. Peak-to-peak linewidths of samples 1, 3, and 133 as a function of temperature between 4.5 and $300 \mathrm{~K}$. For better readability error bars are only included for some of the points.

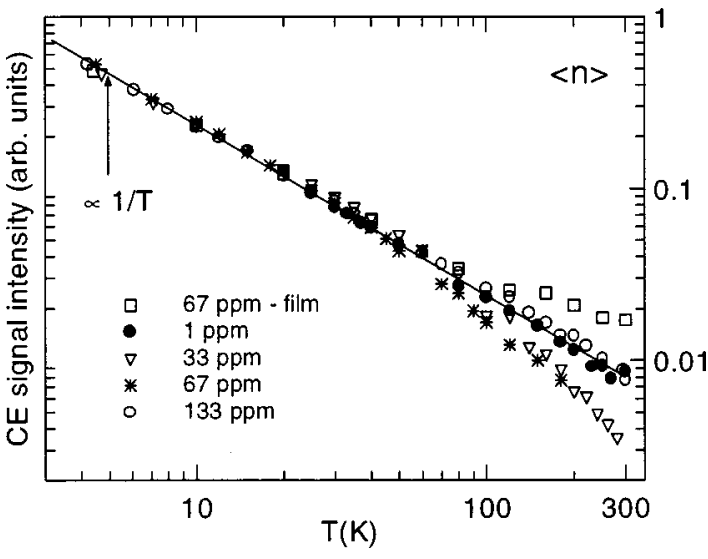

FIG. 6. Temperature dependence of CE signal intensity for powder samples $1,33,67$, and 133 and a thin film of sample 67 between 4.5 and $300 \mathrm{~K}$. Intensity values of the powder samples (except for sample 1) have been corrected for changes in $Q$ with varying temperature. The straight line indicates Curie-like behavior.

corrected for this change in the quality factor. Since reduction of the amount of material inside the cavity reduces the dielectric losses, the $T$ dependence of a thin film of sample 67 (prepared in the same run on glass) is included in Fig. 6.

Powder samples 1 and 133 (which has a relatively small mass of only $18 \mathrm{mg}$ ) exhibit a $1 / T$ behavior in the whole temperature range equivalent to a constant spin density. For the other two samples 33 and 67 the signal intensity decreases faster than expected from the Curie-law at higher temperatures $(T>100 \mathrm{~K})$. In case of sample 33 this deviation is at most a factor of 3 at $300 \mathrm{~K}$. On the other hand, the intensity of the thin film doped with $67 \mathrm{ppm}$ even seems to increase in the high- $T$ region. The apparent increase is probably connected to the uncertainty in determining the area under the absorption curve for the highest temperatures, where the signal intensity is very small for the film sample, the CE line significantly broadens and background contributions are strong.

Under the assumption that the non-Curie behavior of lossy samples (33 and 67) at high temperatures is a result of insufficient experimental corrections when determining the signal intensities, we conclude that the total number (or spin density) of the CE line electrons in general does not change with $T$. However, at the present time additional effects at elevated temperatures cannot be totally ruled out on the basis of the data available. Such an effect would be, for example, the thermal excitation of electrons into states where they give less ESR response thus leading to a deviation from the 1/T behavior.

Having shown that the $\mathrm{CE}$ spin density $N_{S}(\mathrm{CE})$ is largely independent of $T$ we now compare $N_{S}(\mathrm{CE})$ with electron $(n)$ or donor $(N)$ concentrations derived from other experiments. For convenience the values for $N_{S}(\mathrm{CE})$ at $T=40 \mathrm{~K}$ are used, which yields a reasonable signal-to-noise ratio but still avoids saturation effects. The various concentrations $n, N$ are plotted as a function of $N_{S}(\mathrm{CE})$ in Fig. 7, where the dashed line indicates the case when $n, N=N_{S}(\mathrm{CE})$. The solid squares in Fig. 7 represent the phosphorus density $N_{\text {SIMS }}$ obtained by measurements with high mass resolution secondary ion mass spectroscopy (SIMS). $N_{\text {SIMS }}$ turned out to be essentially equal to $N_{S}(\mathrm{CE})$. Using the result that the 


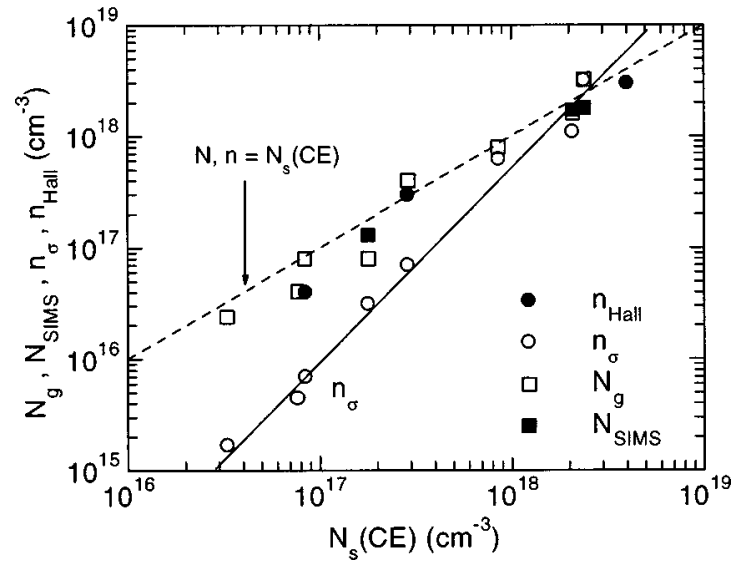

FIG. 7. Electron $(n)$ and donor densities $(N)$ as described in the text plotted versus the $\mathrm{CE}$ spin density $N_{S}(\mathrm{CE})$ at $40 \mathrm{~K}$. The solid line is a guide to the eye and the dotted line denotes the case when $n, N=N_{S}(\mathrm{CE})$.

phosphorus in the gas phase is incorporated into the solid with a build-in ratio of $\mathrm{P}$ to $\mathrm{Si}$ of $0.5,{ }^{47}$ we can further calculate the density of phosphorus atoms $N_{g}$ from the doping ratio in the gas phase according to $N_{g}=0.5 \times$ (gas-phase doping ratio in ppm) $\times 10^{-6} \times 5 \cdot 10^{22} \mathrm{~cm}^{-3}$. Here, the latter quantity is the atomic density of silicon. $N_{g}$ is also plotted in Fig. 7 and agrees remarkably well with the CE spin density between $3 \cdot 10^{16} \mathrm{~cm}^{-3}$ and $2 \cdot 10^{18} \mathrm{~cm}^{-3}$. Hence, $N_{S}(\mathrm{CE})$ is equal to the $\mathrm{P}$ concentration in the solid over 2 orders of magnitude.

For three samples the electron density $n_{\text {Hall }}$ at room temperature was deduced from Hall-effect measurements ${ }^{48}$ and also yields the value of $N_{S}(\mathrm{CE})$ with good accuracy as seen from Fig. 7. This means that the doping efficiency of phosphorus in $\mu c-\mathrm{Si}: \mathrm{H}$ is unity over a wide range of doping levels.

The last quantity shown in Fig. 7 is the electron density $n_{\sigma}$ obtained from the room temperature dark conductivity using $\sigma_{\mathrm{RT}}=n_{\sigma} \cdot e \cdot \mu$ with $\mu \approx 1 \mathrm{~cm}^{2} \mathrm{~V}^{-1} \mathrm{~s}^{-1}$, a typical value for highly doped $\mu c-\mathrm{Si}: \mathrm{H}$ (Refs. 48 and 49) at room temperature. For higher doping levels again $n_{\sigma}=N_{S}(\mathrm{CE})$, whereas both quantities differ at lower phosphorus concentrations.

These results are consistent with the low dangling-bond densities found for all doping levels.

\section{E. Hyperfine interaction}

An electron located at a phosphorus dopant atom in a $P_{4}^{0}$ configuration will, in its ground state, interact with the nucleus thus splitting a single resonance line into a line doublet. In crystalline silicon a hyperfine splitting (i.e., a separation of the respective resonance lines) of $\Delta \mathrm{HFS}=42 \mathrm{G}$ at liquid helium temperature has already been observed in the earliest measurements ${ }^{25}$ (see also Sec. III C).

Although most of our samples are in a doping range where the hf doublet would be the dominant feature in crystalline silicon of similar doping, we never observed a spectrum consisting of two hyperfine lines only. In a certain concentration range $(17-67 \mathrm{ppm})$ two hyperfine lines appear as shoulders in the broad wings of the Lorentzian-type CE cen-

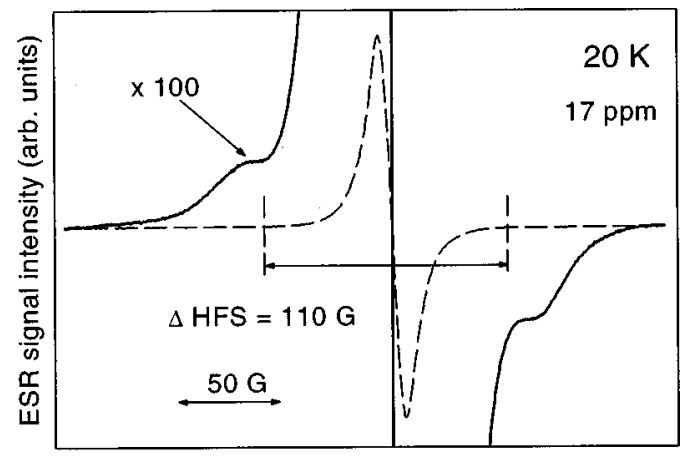

FIG. 8. Dark CW ESR spectrum of sample 17 at $20 \mathrm{~K}$. The magnified curve shows a hyperfine splitting of $110 \mathrm{G}$. Note the enlarged magnetic field range compared to Figs. 1 and 2.

ter line. This is shown in Fig. 8 for sample 17 and a temperature of $20 \mathrm{~K}$. The hyperfine splitting $\Delta \mathrm{HFS}$ is approximately $110 \mathrm{G}$, which is between the values reported for crystalline $(42 \mathrm{G})$ and amorphous silicon $(245 \mathrm{G}) .^{34}$

The hyperfine splitting was best observable in sample 17 , where it could be detected between 4.5 and $70 \mathrm{~K}$. The intensity of the hyperfine doublet at $20 \mathrm{~K}$ is between $2 \%$ and $5 \%$ of the overall signal, and the hf intensity clearly decreases between 20 and $70 \mathrm{~K}$. A detailed study of the temperature dependence was prevented by the weakness of the hyperfine lines as compared to the center line.

In samples with higher doping the hf signal became weaker and, most interestingly, no hyperfine doublet appeared at all in the lightly doped samples. In the latter case, observation of a hf signal of several percent intensity might be impossible due to a poorer signal-to-noise ratio and additional contributions of defect states, which have a similar spin density as the CE line and cannot be totally saturated "away" even at high power levels.

\section{F. Conductivity}

The conductivity of the $\mu c-\mathrm{Si}: \mathrm{H}$ samples recorded in the temperature range between 4.5 and $300 \mathrm{~K}$ is shown in an Arrhenius plot $[\ln (\sigma)$ vs $1000 / T]$ in Fig. 9. For sample 1 and

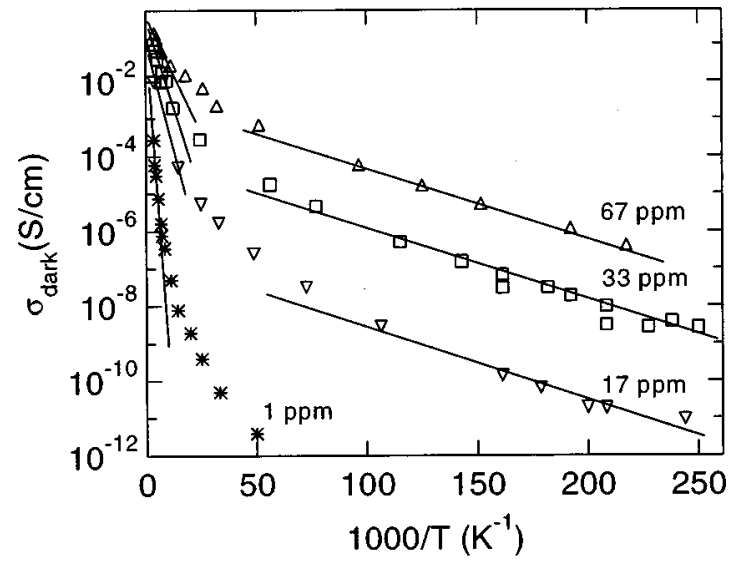

FIG. 9. Dark conductivities $\sigma_{\text {dark }}$ of samples $1,17,33$, and 67 as a function of temperature between 4.5 and $300 \mathrm{~K}$ in an Arrhenius plot. Straight lines indicate regions where approximate values for an activation energy were deduced. 


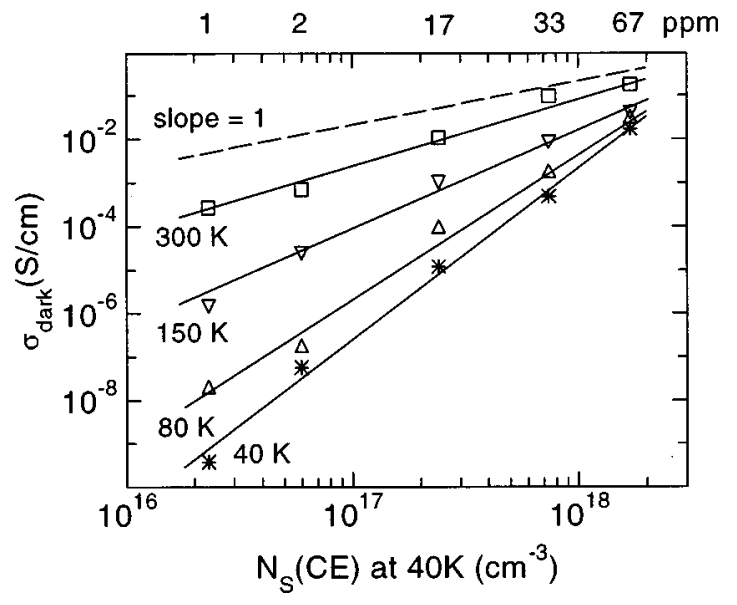

FIG. 10. Dark conductivity $\sigma_{\text {dark }}$ for samples 1 to 67 at various temperatures versus $\mathrm{CE}$ spin density at $40 \mathrm{~K}$. The solid lines are guides for the eye and the dashed line corresponds to slope 1 $\left[\sigma_{\text {dark }} \propto N_{S}(\mathrm{CE})\right]$.

$T<20 \mathrm{~K}$ the conductivity was below our detection limit. All curves exhibit no singly activated behavior, i.e., do not obey a relation $\sigma=\sigma_{0}^{*} \exp \left(-E_{a} / k T\right)$ with a single activation energy $E_{a}$. This is characteristic for microcrystalline material with higher doping levels. ${ }^{5,50-52}$ However, at low temperatures the $\sigma$-vs- $T$ plots can be quite well approximated by a straight line in the temperature region below about $T$ $=20 \mathrm{~K}(1000 / T=50)$. The slope of these lines corresponds to an activation energy of $3.5 \mathrm{meV}$ for all samples. For higher temperatures the range of $E_{a}$ values involved shifts to much higher energies. If one draws lines through the high temperature points, activation energies of $115,35,33$, and 24 meV are obtained for samples $1,17,33$, and 67 , respectively.

Fig. 10 shows a plot of $\sigma_{\text {dark }}$ at various temperatures between 40 and $300 \mathrm{~K}$ versus the spin density $\left(N_{S}\right)$ of the CE line at $40 \mathrm{~K}$. To identify the samples the respective gas-phase doping ratios are also given on the upper $x$ axis. The data exhibit a clear relation between $N_{S}(\mathrm{CE})$ and the conductivity for all temperatures. The solid lines connecting the data points in Fig. 10 are guides for the eye. The dashed line corresponds to a slope of 1 in the double-logarithmic plot, where $\sigma$ would be proportional to $N_{S}(\mathrm{CE})$. It is seen in Fig. 10 that the $\sigma$-vs- $N_{S}$-curves gradually approach such a linear relation with increasing measurement temperature for the conductivity.

\section{DISCUSSION}

In the following, we discuss the above results with regard to their implications for the electronic states in microcrystalline silicon. Our main concern will be the identification and energy position of the respective states of the $\mathrm{CE}$ resonance as a function of temperature and occupation. Additionally, the relation between ESR and transport measurements will be established. In this context, we will also compare our results with data on crystalline silicon and, to some extent, on polycrystalline and porous silicon.

\section{A. $g$ value}

The CE $g$-values found for $\mu c$-Si:H are generally lower than what is reported for crystalline, polycrystalline or po- rous silicon. For temperatures well below room temperature the $g$ values in phosphorus-doped silicon with donor concentrations not larger than several times $10^{18} \mathrm{~cm}^{-3}$ are in the range $1.9985<g<1.9995 .^{9,14,16,29,46,53}$ The highest value of $g=1.9995 \pm 0.0001$ was only recently reported by Young et $a l .{ }^{9}$ as an accurate measurement for the $g$ value of delocalized electrons in the conduction band (CB). It was found at $T=3.5,125$, and $150 \mathrm{~K}$ independent of temperature or doping level. A slight decrease of the $g$ value with rising temperature and increasing doping level was observed by Kodera $^{14,16,46}$ and Stesmans and De Vos. ${ }^{29}$ This was also observed for our $\mu c-\mathrm{Si}: \mathrm{H}$ samples and can be related to the shift of the electron distribution from tail states to conduction-band states at higher temperature or higher doping level.

In polycrystalline silicon (poly-Si) $g$ values of conduction electrons between 1.997 and 1.999 were reported by Hasegawa, Kasajima and Shimizu ${ }^{54}$ for phosphorus-doped CVD amorphous silicon after annealing to $700-750{ }^{\circ} \mathrm{C}$.

Young, Pointdexter, and Gerardi ${ }^{55}$ find the same $g$ value as in $c$-Si of $g=1.9995$ both in $n$ - and in $p$-type porous silicon and attribute it to conduction band electrons in silicon microcrystals. Von Bardeleben et al. ${ }^{56}$ observe ESR signals of optically excited free electrons in what they call quantum confined Si nanostructures with orientation-dependent $g$ values of $g_{\perp}=1.9991$ and $g_{\|}=1.9986$.

The $g$ values obtained in microcrystalline silicon by other authors are 1.997 in phosphorus-doped, ${ }^{57} 1.9983$ in nitrogen-doped, ${ }^{7} 1.9981$ in intrinsic $\mu c$-Si:H samples annealed to $400{ }^{\circ} \mathrm{C}$ (Ref. 12) and $g=1.998$ in intrinsic $\mu c-\mathrm{Si}: \mathrm{H}$ under light illumination ${ }^{8}$ in good agreement with our own data.

In the past, a lot of work has been done to calculate theoretically the expected $g$ shifts in crystalline semiconductors such as silicon (see, e.g., Ref. 58 for a review), but similar calculations for microcrystalline silicon have not been performed so far. The $g$ value is influenced by the wave functions of the corresponding electronic states and by local strains within the material, for example produced by an impurity atom like phosphorus. In the case of conduction-band electrons, the $g$ value depends on the band structure and the respective Bloch functions, and it will even be different for electrons near or far away from a band degeneracy. ${ }^{59}$ Local strains are expected to vary in microcrystalline and crystalline silicon, and distinctive differences have been found experimentally for the impurity wave functions in both materials (see Sec. IVE and VD). Hence, a variation in the respective electronic $g$ values of $\mu c-\mathrm{Si}: \mathrm{H}$ and $c-\mathrm{Si}$ is not surprising. However, there is no simple picture which would allow a quantitative prediction of this difference.

The decrease in $g$ value with phosphorus concentration $N_{D}$ in $\mu c-\mathrm{Si}: \mathrm{H}$ is possibly connected to the formation of donor clusters at higher dopant density leading to a partial delocalization of the electrons within the cluster. One expects that with increasing donor concentration the $g$ value approaches the one of electrons in the conduction band. ${ }^{26} \mathrm{~A}$ good estimate for the latter is $g=1.9963$ (the smallest observed $g$ value) of sample 133 at $300 \mathrm{~K}$ (when almost all of the electrons are thermally excited into the $\mathrm{CB}$ ) and, consequently, approaching the $\mathrm{CB} g$ value with increasing donor concentration means a decrease in $g$ value. 


\section{B. Linewidth}

The CE linewidth $\Delta H_{p p}$ in $\mu c-\mathrm{Si}: \mathrm{H}$ is much larger than in $c$-Si, while similar values are reported for poly-Si. ${ }^{54}$ It increases with doping and temperature for $T>30 \mathrm{~K}$, but around $T=30 \mathrm{~K}$ a distinct minimum appears in samples with doping levels of more than $33 \mathrm{ppm}$.

In dilute spin systems, where the spin-lattice relaxation time $T_{1}$ acts as an average lifetime of the spin states $\left(T_{1}\right.$ $\left.\approx T_{2}\right)$, the linewidth would be given by $\Delta H=1 /\left(\gamma T_{1}\right)$ with $\gamma=g_{e} \mu_{B} / \hbar$. Using $T_{1}=10^{-5} \mathrm{~s}$ at $30 \mathrm{~K}$ for sample 1 (Ref. 17) would result in a linewidth of only $6 \mathrm{mG}$. Hence, around the position of the minimum the large value of $\Delta H_{p p}(8 \mathrm{G}$ for all samples) is caused by a distribution of $g$ values and unresolved hyperfine structure rather than a short spin-lattice relaxation time. However, the data by Malten, Müller, and Finger ${ }^{17}$ exhibit a steep decrease of $T_{1}$ in $\mu c$-Si:H for $T$ $>60 \mathrm{~K}$. Therefore, we attribute the strong increase in line broadening with rising temperature for $T>60 \mathrm{~K}$ to the corresponding decrease in spin-lattice relaxation time. A steady increase in linewidth for temperatures between liquid helium and room temperature has also been found in the earliest ESR studies by Portis, Kip, and $\mathrm{Kittel}^{13}$ and later by various other authors. ${ }^{16,60}$

An interesting feature is the appearance of the minimum (Fig. 5), which was also reported for crystalline silicon in samples with donor concentrations of less than 3.5 $\times 10^{18} \mathrm{~cm}^{-3}$ (Refs. 21, 29, 60-63). This minimum was mostly explained by motional or exchange narrowing of the resonances, which becomes less effective with decreasing temperature. In earlier works by Maekawa and Kinoshita ${ }^{21}$ the increase of linewidth with decreasing temperature was interpreted as a change in the correlation frequency of the exchange interaction due to reduced hopping rates of electrons between neighboring sites at lower temperatures. On the other hand Murayama, Clark, and Sanny ${ }^{63}$ explain their data in a picture where spins (donor electrons) are randomly located and coupled with each other via antiferromagnetic exchange interaction. The spins of such a system tend to condense into singlet ground states of donor clusters as $T$ is lowered, ${ }^{64}$ which reduces the effective exchange experienced by the remaining unpaired spins, and the narrowing effect becomes smaller.

Even without deciding which of these pictures correctly describes the situation in $\mu c-\mathrm{Si}: \mathrm{H}$ we assume the presence of some kind of narrowing effect for the CE line. In a certain temperature region above $30 \mathrm{~K}$, where a large fraction of the electrons is still located at donor sites or in conduction band tail states, such narrowing effects lead to a somewhat smaller linewidth than would be obtained without them. As one goes to very low temperatures, the narrowing effect becomes weaker resulting in an overall increase in linewidth and leading to the observed minimum.

The absence of the minimum in lightly doped material may well be explained in both models. In samples with lower $\mathrm{CE}$ spin densities the distance between interacting spins becomes too large to allow for effective exchange interaction or for sufficiently rapid hopping motion. This can be checked by calculating the average distance $r_{D}$ between the electrons contributing to the $\mathrm{CE}$ line using the expression $r_{D}$ $=\left(4 \pi / 3 * N_{S}\right)^{-1 / 3}$ where $N_{S}=N_{S}(\mathrm{CE})$ is the observed CE spin density. This leads to values $r_{D}$ of 65,52 , and $48 \AA$ for samples 33, 67, and 133, respectively. On the other hand, for sample 1 (with a spin density that is about a factor of 100 smaller than in sample 133) $r_{D}$ equals $193 \AA$ and is too large for exchange averaging to be expected. Furthermore, a comparison of the hopping frequencies estimated from the ratio of the factor $\exp \left(-2 r_{D} / a\right)$, yields frequencies lower by 10 orders of magnitude for sample 1 as compared to sample 133. For the parameter $a$ we used a value of $12 \AA$, which is the effective Bohr radius of the impurity wave function in $n$-type $\mu c-\mathrm{Si}: \mathrm{H}$ as estimated from hyperfine interaction data (Sec. V D).

The increase in linewidth with doping at higher temperatures is in accordance with the decreasing spin-lattice relaxation times with increasing doping level as deduced from saturation measurements (not shown). A similar broadening of the resonance line with doping has also been found in early studies on heavily doped (>1000 ppm) $\mu c$-Si:H (Ref. 57) with a linewidth of about $18 \mathrm{G}$ at liquid nitrogen temperature (as compared to $12 \mathrm{G}$ in the present study, see Fig. 5).

\section{Magnetic susceptibility}

For comparison we first summarize the situation in $n$-type crystalline silicon. A Curie-like susceptibility was found for the electrons located at the donor atoms in weakly phosphorus doped $n$-type crystalline silicon. ${ }^{21,26}$ Also in the earliest spin resonance measurements on $n$-type $\mathrm{Si}$ (Ref. 13) with $N_{D}=1-2 \times 10^{18} \mathrm{~cm}^{-3}$ Curie behavior for temperatures between 80 and $300 \mathrm{~K}$ was observed. A deviation from the Curie law at $4.2 \mathrm{~K}$ was interpreted as the change from Curie to Pauli paramagnetism.

To account for the two general types of magnetic behavior some authors describe their experimental susceptibility data with a two-component model, consisting of electrons following a Curie or Curie-Weiss law [Eq. (1)] and electrons with a Pauli-spin-susceptibility [Eq. (2) $].{ }^{26,61}$ In these works the concentrations of the two different types of electrons are obtained as results of numerical fits to the underlying model and are independent of temperature.

In Fig. 6, Curie-like behavior of the conduction electron resonance in $\mu c$-Si:H was demonstrated between liquid helium and room temperature (with deviations for some samples at the highest temperatures). To check for which temperature region a Pauli-like susceptibility component could be theoretically expected, we calculate the degeneracy temperature $T_{\text {deg }}$ below which an electron gas becomes degenerate:

$$
T_{\text {deg }}=\frac{h^{2}}{8 k m^{*}} \cdot(3 / \pi)^{2 / 3} \cdot n^{2 / 3} .
$$

In Eq. (4) $n$ is the electron concentration and $m^{*}$ the effective mass of the electrons, which we take as the densityof-state effective mass $m^{*}=1.08 \cdot m_{e}$ (Ref. 46) $\left(m_{e}\right.$ $=$ free electron mass, $h=$ Planck's constant). For our range of electron concentrations $N_{S}(\mathrm{CE})$ between $3 \times 10^{16}$ and $2.5 \times 10^{18} \mathrm{~cm}^{-3}$ we obtain degeneracy temperatures between 3.8 and $72 \mathrm{~K}$, respectively. If the electrons contributing to the conduction electron resonance formed a free electron gas, 
one would expect degeneracy and an onset of Pauli paramagnetism for temperatures below $72 \mathrm{~K}$ for sample 133. However, in $\mu c-\mathrm{Si}: \mathrm{H}$ we find that a $1 / T$ law is obeyed very well down to $4.5 \mathrm{~K}$, and there is no Pauli-like susceptibility component in our $\chi(T)$ data neither in form of a transition Curie $\rightarrow$ Pauli nor as a superposition Curie + Pauli. It should be investigated in future studies, if at still lower temperatures $(T<4.2 \mathrm{~K})$ and/or at higher dopant concentrations Pauli paramagnetism can be observed in $\mu c-\mathrm{Si}: \mathrm{H}$.

\section{Hyperfine interaction}

The simplest approximation to describe the ground-state donor wave function $\psi$ in phosphorus-doped crystalline silicon is a spherically symmetric $s$-like wave function, whose spatial extent is given in terms of the Bohr or fall-off radius $a$. However, the actual electron density distribution around a donor in $c$-Si deviates substantially from a spherically symmetric form (see, e.g., the calculations by Ivey and Mieher $^{65}$ ). As no comparable experimental data exist for $\mu c$-Si:H, we follow the procedure given by Stutzmann, Biegelsen, and Street $^{34}$ (who use a spherically symmetric $\psi$ ) in order to obtain an approximate value for the effective Bohr radius $a$ from the magnitude of the hyperfine splitting $\Delta$ HFS. In terms of the hyperfine splitting the isotropic hyperfine interaction $A$ [see Eq. (3) in Sec. III C] is given by $A$ $=g_{e} \mu_{B} \Delta$ HFS and hence,

$$
\left|\psi_{e}(r=0)\right|^{2} \propto \Delta \mathrm{HFS} .
$$

For the effective Bohr radius $a$ of the donors the following relation holds

$$
a \propto\left(\left|\psi_{e}(r=0)\right|^{2}\right)^{-1 / 3} .
$$

Therefore, we can directly relate the values of the hyperfine splittings in $\mu c-\mathrm{Si}: \mathrm{H}$ and crystalline silicon to their effective Bohr radii. Using the value of $a=16.7 \AA$ in $c$-Si (Ref. 34) we obtain $a(\mu c-\mathrm{Si}: \mathrm{H})=12 \AA$. Thus the electrons bound in the assumably $s$-like donor ground state in $\mu c-\mathrm{Si}: \mathrm{H}$ are stronger localized at the nucleus than in crystalline material, but the localization is weaker than in amorphous silicon $[a(a-\mathrm{Si}: \mathrm{H})=10 \AA]$.

The intensity of the hyperfine interaction does not scale with phosphorus concentration in contrast to other ESR parameters like $g$ value, CE intensity, and linewidth. At this point, it is too early for a conclusive interpretation of the behavior of the hyperfine interaction in the different temperature and doping regimes, but some hints may be obtained from the assumed density-of-states distribution in $\mu c-\mathrm{Si}: \mathrm{H}$ and a comparison with crystalline silicon. To this end, it is convenient to treat the cases of high- and lowdoping levels separately.

For higher doping levels the nonobservability of hf interaction is in accordance with what is known from $c$-Si (see Sec. III C) where it results from impurity cluster formation and possibly excitation into an impurity band. In addition, when analyzing the low-temperature conductivity data for $\mu c$-Si:H samples with higher doping levels in the next section, it will be suggested that transport in $\mu c-\mathrm{Si}: \mathrm{H}$ at low $T$ proceeds via hopping between ionized and neutral donor sites. If the hopping frequency for such a process exceeds the frequency $\omega=1 / T_{1}$ associated with the experimental values of the spin-lattice relaxation time $T_{1},{ }^{17}$ such an electron will successively observe positive and negative values of the hyperfine field, and the hyperfine interaction will be effectively averaged to zero.

The more puzzling result is the nonobservation of hf interaction at low doping, which is in contrast to what one expects from the comparison with crystalline silicon data. This has important consequences for the nature and location of the CE states in $\mu c-\mathrm{Si}: \mathrm{H}$. Two cases have to be considered: (i) the majority of the electrons seen as CE line in ESR are not located at their phosphorus donors at all or (ii) the electrons are in fact bound to the phosphorus atoms, but for some reason no hyperfine interaction can be detected. (i) If electrons are not located at their donor sites, they could possibly be excited into the conduction band, have got trapped by deep defects (as observed in polycrystalline silicon ${ }^{54}$ ) or occupy conduction-band tail states. The first possibility is readily ruled out, as for temperatures below $40 \mathrm{~K}$ only less than $10 \%$ of the donor electrons will have been activated into the conduction band ${ }^{38}$ assuming the same donor binding energy as in $c$-Si $(45 \mathrm{meV}){ }^{66}$ Furthermore, most of the doping induced electrons cannot be trapped at defects, as the number of $\mathrm{CE}$ electrons is equal to the number of incorporated donors over two orders of magnitude (Sec. IV D). Hence, it seems very likely that electrons occupy tail states in the conduction-band tails of the silicon crystallites. The energy range below the conduction band of such tail states overlaps with the binding energy of the phosphorus donor electrons (45 meV in $c$-Si). Thus, electron transfer between donor and tail states is likely to occur and an occupation of tail states might be even more favorable for electrons, as a certain fraction of tail states has energies lower than the donor energy. (ii) Like in samples with higher doping levels, cluster formation or averaging out of the hyperfine interaction as a result of hopping processes have to be considered. The former cannot be ruled out, but we note that this would be in contrast to $c$-Si where clustering begins to influence the hf spectrum only at considerably higher doping levels. An averaging effect due to hopping between donor sites can be excluded as the hopping frequencies for such processes estimated from the average interdonor distance $r_{D}$ (193 $\AA$ for a CE spin density of $3 \times 10^{16} \mathrm{~cm}^{-3}$ in sample 1) are too low and hopping motion would not be rapid enough to lead to an averaging effect. Finally, an excitation of donor electrons into excited states of phosphorus (where they show no hf interaction) has to be considered. This is again ruled out by Lépine's calculations, ${ }^{38}$ which show that at $T<40 \mathrm{~K}$ in fact most of the electrons are in the hyperfine-active ground state $A_{1}$.

In summary, possible explanations for the low-hf intensities are occupancy of conduction band tail states instead of donor states, formation of clusters of two or more donor atoms and the effect of averaging out the hf interaction by rapid hopping motion of electrons between adjacent donor sites. The latter process only applies to samples with higher doping levels.

\section{E. Electronic transport}

From the agreement between dopant and carrier densities deduced from the different methods with the ESR spin den- 
sities $N_{S}$ of the CE resonance (Sec. IV D) it is obvious that both electronic transport and electron spin resonance are governed by the excess electrons introduced via the doping process. We suggest the following mechanisms of electronic transport in the low- and high-temperature regions, respectively.

$20 \mathrm{~K}<T<300 \mathrm{~K}$ : At elevated temperatures carrier transport proceeds in the conduction band, into which the electrons were excited from shallow donor states (or tail states). This assumption is just a consequence of the agreement between the phosphorus density and the electron density $n_{\sigma}$ calculated from $\sigma=n_{\sigma} \cdot e \cdot \mu$ for high donor concentrations. At room temperature all of the donors are ionized, the thermal energy is high enough to have a sufficient number of allowed percolation paths and transport takes place by quasifree electrons in the $\mathrm{CB}$ moving along these paths, crossing lower barriers and avoiding high ones. The decrease of the conductivity with a lowering of $T$ is ascribed both to the decreasing number of electrons excited to the $\mathrm{CB}$ and to the increased length of percolation paths for the carriers. ${ }^{49,67}$

The non-Arrhenius behavior in this temperature range can be interpreted as a distribution of activation energies as a result of varying barrier heights between crystalline regions and the thermal excitation out of tail states with varying energy positions below the conduction band. With decreasing doping level, $n_{\sigma}$ deviates more and more from $N_{S}(\mathrm{CE})$. The main reason for that is the decrease in carrier mobility going to lower doping levels as found in recent Hall-effect measurements. ${ }^{48}$ Furthermore, some of the donor electrons might have been trapped by deep defects or tail states, although the number of trapped electrons has to be relatively small to be compatible with the high CE spin density. This decrease in $\mu$ at lower doping levels is remarkably different from crystalline silicon where the carrier mobility usually increases when the number of dopants is reduced as a result of a reduction in ionized impurity scattering.

$T<20 \mathrm{~K}$ : For the lowest temperatures a temperatureactivated behavior of the conductivity was found (Sec. IV F) with an activation energy $E_{a}$ of $3.5 \mathrm{meV}$ for all samples (17, 33 , and 67). In crystalline silicon at low temperatures an activated transport process with an activation energy $\Delta E_{\text {hop }}$ of several meV is well known and referred to as nearestneighbor hopping. It takes place in crystalline semiconductors between donor or acceptor sites. A necessary condition is the simultaneous presence of neutral and ionized dopant states, which can be accomplished by partial compensation. ${ }^{68,69}$ Since an activation energy (of $3.5 \mathrm{meV}$ ) is also observed for $T<20 \mathrm{~K}$ in $\mu c-\mathrm{Si}: \mathrm{H}$, an applicability to our case seems appropriate. In $\mu c-\mathrm{Si}: \mathrm{H}$ partial ionization of donor atoms can be achieved by electron trapping in defect states or conduction-band tail states. In both cases the ionized donor is in the direct vicinity of a state that has taken up an additional electron. The resulting repulsive Coulomb potential seen by a hopping electron leads to the observed activation energy. In crystalline semiconductors with low compensation the (hopping) mobility at low $T$ is given as ${ }^{70}$

$$
\sigma_{\text {hop }}=\sigma_{00} \cdot \exp \left(-\frac{2 r_{c}}{a}\right) \cdot \exp \left(-\frac{\Delta E_{\text {hop }}}{k T}\right)
$$

where $r_{c}$ is essentially the distance between hopping centers (donors) and is given by $r_{c}=(0.865 \pm 0.015) \cdot N_{D}^{-1 / 3}$ with donor density $N_{D}$ (Ref. 71) and $a$ denotes again the effective Bohr radius of the donor electron wave function [see Eq. (3)]. A calculation of $a$ from an extrapolation $(T \rightarrow \infty)$ of the conductivity values for samples 17, 33, and 67 (Fig. 9) yields effective Bohr radii between 11 and $14 \AA$, which compare very well to the value of $12 \AA$ obtained from the hyperfine splitting in Sec. V D. Furthermore, experimental works on doped $p$ - and $n$-type crystalline silicon yield activation energies $\Delta E_{\text {hop }}$ around $5 \mathrm{meV}$ very similar to the value of 3.5 meV found in our samples. ${ }^{72,73}$

As the conductivity decreases exponentially with increasing distance between hopping sites (interdonor distance) it drops below the detection limit in lightly doped $\mu c-\mathrm{Si}: \mathrm{H}$ material at low temperatures.

In conclusion, the low-temperature conductivity in microcrystalline silicon can be described by the well-established model of nearest-neighbor hopping between neutral and ionized donors as adopted from crystalline transport theory. However, the hopping process can involve both donor and conduction band tail states.

\section{SUMMARY AND CONCLUSIONS}

The doping and temperature dependence of the conduction electron ESR signal (CE) and the conductivity in a series of $n$-type $\mu c-\mathrm{Si}: \mathrm{H}$ samples has been studied. The results allow to identify the origin of the $\mathrm{CE}$ resonance in the various temperature and doping regimes and to establish the relationship between ESR and transport data.

The CE resonance in $\mu c-\mathrm{Si}: \mathrm{H}$ can be related to doping induced excess electrons. The CE spin density is equal to the donor density over a doping range from $3 \times 10^{16}$ to 2 $\times 10^{18} \mathrm{~cm}^{-3}$. The temperature and doping dependence of the $\mathrm{CE}$ resonance line parameters is similar to crystalline silicon, but the absolute values show some distinct differences (smaller $g$ values, larger linewidth).

The single-line CE resonance is prominent in the entire doping and temperature range. The hyperfine doublets of electrons located at $\mathrm{P}$ donor atoms can be clearly distinguished only at intermediate doping levels, where they account at most for $5 \%$ of the signal. From the hyperfine splitting of $\Delta \mathrm{HFS}=110 \mathrm{G}$ a value of $a=12 \AA$ for the effective Bohr radius of the donor electron wave function is derived, compared to $16.7 \AA$ in $c$ - $\mathrm{Si}$ and $10 \AA$ in amorphous silicon. This means that the degree of localization of the donor electrons is between $c$-Si and $a-\mathrm{Si}: \mathrm{H}$. Similar values also follow from the low-temperature conductivity data.

The absence of visible hyperfine interaction for the highest doping levels is in accordance with observations in crystalline silicon, where it is explained by donor clustering and averaging of the hyperfine interaction as a result of the hopping motion of electrons between phosphorus donors. As the main reason for the lack of hyperfine interaction at low doping levels (in contrast to $c$-Si), we suggest the occupancy of conduction-band tail states.

At low temperatures the electrons giving rise to the $\mathrm{CE}$ resonance are either localized at their donor sites or occupy conduction band tail states within the crystalline grains. At $T<20 \mathrm{~K}$ transport proceeds via hopping between neutral and 
ionized donors and/or conduction-band tail states. This kind of transport is characterized by a single activation energy of $3.5 \mathrm{meV}$ in $\mu c-\mathrm{Si}: \mathrm{H}$.

With rising temperature an increasing number of electrons is excited into delocalized conduction-band states, but at any temperature the charge carriers are nondegenerate for the doping range investigated, leading to a Curie-like behavior of the susceptibility (with deviations for some of the samples at the highest temperatures). The electrons in the conduction band are still observed with ESR, but the transition to delocalized states is accompanied by appreciable line broadening and a decrease in $g$ value. The line broadening is a result of the strong decrease in spin-lattice relaxation time with rising temperature. A minimum in the linewidth-vs- $T$-data around $30 \mathrm{~K}$ is interpreted-like in crystalline silicon-with a decreasing exchange narrowing effect as one goes to low temperatures.
At higher temperatures transport cannot be described by a single-activation energy. Here, the conduction process is understood in terms of a percolation model where carriers move in an interconnected network with variable barrier heights between adjacent regions, a mechanism completely different from crystalline silicon.

\section{ACKNOWLEDGMENTS}

We greatly benefited from numerous discussions with $\mathrm{H}$. Overhof. We further thank D. Mihelcic and M. Kloza for their comments on the cavity $Q$ factor, J. Wolff and A. Lambertz for sample preparation and technical assistance, $\mathrm{H}$. Holzbrecher for SIMS, and N. Harder for Hall effect measurements. This work was supported by the Bundesministerium für Bildung, Wissenschaft, Forschung und Technologie.
${ }^{1}$ H. Meier, P. Torres, R. Platz, S. Dubail, U. Kroll, J. A. Anna Selvan, N. Pellaton Vaucher, Ch. Hof, D. Fischer, H. Keppner, A. Shah, K.-D. Ufert, P. Giannoules, and J. Koehler, Amorphous Silicon Technology, edited by M. Hack, E. A. Schiff, S. Wagner, A. Matsuda, and R. Schopp, MRS Symposia Proceedings No. 420 (Materials Research Society, Pittsburgh, 1996), p. 3.

${ }^{2}$ K. Yamamoto, T. Suzuki, M. Yoshimi, and A. Nakajima, Jpn. J. Appl. Phys., Part 2 36, L569 (1997).

${ }^{3}$ M. Luysberg, P. Hapke, R. Carius, and F. Finger, Philos. Mag. A 75, 31 (1997).

${ }^{4}$ L. Houben, M. Luysberg, P. Hapke, R. Carius, F. Finger, and H. Wagner, Philos. Mag. A 77, 1447 (1998).

${ }^{5}$ F. Finger, C. Malten, P. Hapke, R. Carius, R. Flückiger, and H. Wagner, Philos. Mag. Lett. 70, 247 (1994).

${ }^{6}$ C. Malten, F. Finger, P. Hapke, T. Kulessa, C. Walker, R. Carius, R. Flückiger, H. Wagner, Microcrystalline and Nanocrystalline Semiconductors, edited by L. Brus, M. Horose, R. W. Collins, F. Kock, and C. C. Tsai, MRS Symposia Proceedings No. 358 (Materials Research Society, Pittsburgh, 1995), p. 757.

${ }^{7}$ T. Ehara, Appl. Surf. Sci. 113/114, 126 (1997).

${ }^{8}$ M. Kondo, T. Nishimiya, K. Saito, and A. Matsuda, J. Non-Cryst. Solids 227-230, 1031 (1998).

${ }^{9}$ C. F. Young, E. H. Pointdexter, G. J. Gerardi, W. L. Warren, and D. J. Keeble, Phys. Rev. B 55, 16245 (1997).

${ }^{10}$ E. Holzenkämpfer, F. W. Richter, J. Stuke, and U. Voget-Grote, J. Non-Cryst. Solids 32, 327 (1979).

${ }^{11} \mathrm{P}$. Hapke and A. Mück (private communication).

${ }^{12}$ C. Lips, P. Kanschat, D. Will, C. Lerner, and W. Fuhs, J. NonCryst. Solids 227-230, 1021 (1998).

${ }^{13}$ A. M. Portis, A. F. Kip, and C. Kittel, Phys. Rev. 90, 988 (1953).

${ }^{14}$ H. Kodera, J. Phys. Soc. Jpn. 19, 915 (1964).

${ }^{15}$ J. Müller, F. Finger, C. Malten, and H. Wagner, J. Non-Cryst. Solids 227-230, 1026 (1998).

${ }^{16}$ H. Kodera, J. Phys. Soc. Jpn. 27, 1197 (1969).

${ }^{17}$ C. Malten, J. Müller, and F. Finger, Phys. Status Solidi B 201, R15 (1997).

${ }^{18}$ F. Finger, J. Müller, C. Malten, and H. Wagner, Philos. Mag. B 77, 805 (1998)

${ }^{19}$ G. Feher and R. C. Fletcher, Bull. Am. Phys. Soc. 1, 125 (1956).

${ }^{20}$ G. Feher and E. A. Gere, Phys. Rev. 114, 1245 (1959).
${ }^{21}$ S. Maekawa and N. Kinoshita, J. Phys. Soc. Jpn. 20, 1447 (1965).

${ }^{22}$ H. Nagashima and H. Yamasaki, J. Phys. Soc. Jpn. 41, 711 (1976).

${ }^{23}$ J. Müller, F. Finger, C. Malten, and H. Wagner, in Advances in Microcrystalline and Nanocrystalline Semiconductors-1996, edited by R. W. Collins et al., MRS Symposia Proceedings No. 452 (Materials Research Society, Pittsburgh, 1997), p. 827.

${ }^{24}$ C. Malten, Ph.D. thesis, Technical University Aachen, 1996.

${ }^{25}$ R. C. Fletcher, W. A. Yager, G. L. Pearson, and F. R. Merritt, Phys. Rev. 95, 844 (1954).

${ }^{26}$ P. R. Cullis and J. R. Marko, Phys. Rev. B 11, 4184 (1975).

${ }^{27}$ G. Feher, R. C. Fletcher, and E. A. Gere, Phys. Rev. Lett. 100, 1784 (1955).

${ }^{28}$ E. Mooser, Phys. Rev. 100, 1589 (1955).

${ }^{29}$ A. Stesmans and G. De Vos, Phys. Rev. B 34, 6499 (1986).

${ }^{30} \mathrm{~J}$. Werner and M. Peisl, in Microscopic Identification of Electronic Defects in Semiconductors, edited by N. M. Johnson, S. G. Bishop, and G. D. Watkins, MRS Symposia Proceedings No. 46 (Materials Research Society, Pittsburgh, 1985), p. 575.

${ }^{31}$ J. Werner and M. Peisl, Phys. Rev. B 31, 6881 (1985).

${ }^{32}$ D. Jousse, S. L. Delage, and S. S. Iyer, Philos. Mag. B 63, 443 (1991).

${ }^{33}$ J. H. Werner, in Polycrystalline Semiconductors, edited by J. H. Werner, H. J. Möller, and H. P. Strunk, Springer Proceedings in Physics Vol. 35 (Springer, Berlin, 1989), p. 345.

${ }^{34}$ M. Stutzmann, D. K. Biegelsen, and R. A. Street, Phys. Rev. B 35, 5666 (1987).

${ }^{35}$ N. M. Atherton, Principles of Electron Spin Resonance (Prentice Hall, New York, 1993).

${ }^{36}$ W. Pauli, Z. Phys. 41, 81 (1927).

${ }^{37}$ E. Fermi, Z. Phys. 60, 320 (1930).

${ }^{38}$ D. J. Lépine, Phys. Rev. B 2, 2429 (1970).

${ }^{39}$ W. Kohn, in Solid State Physics, edited by F. Seitz and D. Turnbull (Academic, New York, 1957), Vol. 5, p. 257

${ }^{40}$ C. P. Slichter, Phys. Rev. 99, 479 (1955).

${ }^{41}$ J. M. Winter, Phys. Soc. Jpn. 21, Suppl., 213 (1966).

${ }^{42}$ K. Morigaki and S. Maekawa, J. Phys. Soc. Jpn. 32, 462 (1972).

${ }^{43}$ M. Morooka, M. Tokita, T. Kato, and I. Tsurumi, Jpn. J. Appl. Phys., Part 1 33, 1872 (1994).

${ }^{44}$ F. J. Dyson, Phys. Rev. 98, 349 (1955). 
${ }^{45}$ H. Kodera, J. Phys. Soc. Jpn. 28, 89 (1970).

${ }^{46}$ H. Kodera, J. Phys. Soc. Jpn. 21, 1040 (1966).

${ }^{47}$ J. Müller, Ph.D. thesis, Technical University Aachen, 1998.

${ }^{48}$ R. Carius, J. Müller, F. Finger, N. Harder, P. Hapke, in Proceedings of the 10th International School on Condensed Matter Physics, Varna, Bulgaria, 1998 (World Scientific, Singapore, 1999).

${ }^{49}$ U. Backhausen, R. Carius, F. Finger, P. Hapke, U. Zastrow, and H. Wagner, in Advances in Microcrystalline and Nanocrystalline Semiconductors-1996 (Ref. 23), p. 833.

${ }^{50}$ J. Dubois, G. Willeke, K. Prasad, O. Blenk, A. Shah, E. Bucher, in Proceedings of the 11th European Community Photovoltaic Solar Energy Conference, edited by L. Guimarães, W. Palz, C. De Reyff, H. Kiess, and P. Helm (Harwood Academic Publishers, Chur, Switzerland, 1993), p. 718.

${ }^{51}$ F. Finger, R. Carius, P. Hapke, K. Prasad, and R. Flückiger, in Microcrystalline Semiconductors: Materials Science \& Devices, edited by P. M. Fauchet et al., MRS Symposia Proceedings No. 283 (Materials Research Society, Pittsburgh, 1993), p. 471.

${ }^{52}$ P. Hapke, U. Backhausen, R. Carius, F. Finger, and S. Ray, in Compound Semiconductor Electronics and Photonics, edited by R. J. Shul et al., MRS Symposia Proceedings No. 421 (Materials Research Society, Pittsburgh, 1996), p. 789.

${ }^{53}$ G. Feher, Phys. Rev. 114, 1219 (1959).

${ }^{54}$ S. Hasegawa, T. Kasajima, and T. Shimizu, J. Appl. Phys. 50, 7256 (1979).

${ }^{55}$ C. E. Young, E. H. Pointdexter, and G. J. Gerardi, J. Appl. Phys. 81, 7468 (1997).

${ }^{56}$ H. J. von Bardeleben, C. Ortega, A. Grosman, V. Morazzani, J. Siejka, and D. Stievenard, J. Lumin. 57, 301 (1993).
${ }^{57}$ S. Hasegawa, S. Narikawa, and Y. Kurata, Philos. Mag. B 48, 431 (1983).

${ }^{58}$ Y. Yafet, in Solid State Physics, edited by F. Seitz and D. Turnbull (Academic, New York, 1953), Vol. 14, p. 1

${ }^{59}$ R. J. Elliott, Phys. Rev. 96, 266 (1954).

${ }^{60}$ J. D. Quirt, Phys. Rev. B 7, 3842 (1973).

${ }^{61}$ H. Ue, S. Maekawa, Phys. Rev. B 3, 4232 (1971)

${ }^{62}$ G. Lancaster, J. A. Van Wyk, and E. E. Schneider, Proc. Phys. Soc. London 84, 19 (1964).

${ }^{63}$ C. T. Murayama, W. G. Clark, and J. Sanny, Phys. Rev. B 29, 6063 (1984).

${ }^{64}$ R. N. Bhatt and P. A. Lee, Phys. Rev. Lett. 48, 344 (1982).

${ }^{65}$ J. L. Ivey and R. L. Mieher, Phys. Rev. B 11, 822 (1975), in particular Fig. 15.

${ }^{66}$ G. Picus, E. Burstein, and B. Henvis, J. Phys. Chem. Solids 1, 65 (1956).

${ }^{67}$ R. Carius, F. Finger, M. Luysberg, P. Hapke, U. Backhausen, in Future Directions in Thin Film Science and Technology, edited by J. M. Marshall, N. Kirov, A. Vavrek, J. M. Maud (World Scientific, Singapore, 1997), p. 11.

${ }^{68}$ N. F. Mott, Can. J. Phys. 34, 1356 (1956).

${ }^{69}$ E. M. Conwell, Phys. Rev. 103, 51 (1956).

${ }^{70}$ K. W. Böer, Survey of Semiconductor Physics (Van Nostrand Reinold, New York, 1990), p. 900.

${ }^{71}$ J. A. McInnes and P. N. Butcher, Philos. Mag. B 39, 1 (1979).

${ }^{72}$ K. R. Atkins, R. Donovan, and R. H. Walmsley, Phys. Rev. 118, 411 (1960).

${ }^{73}$ J. A. Chroboczek, Noncrystalline Semiconductors (CRC, Boca Raton, FL, 1987), Vol. III, p. 109, and references therein. 